\title{
Bioengineering mini functional thymic units with EAK16-II/EAKIIH6 self-assembling
}

\section{hydrogel}

Asako Tajima ${ }^{1}$, Wen Liu르, Isha Pradhan ${ }^{1}$, Suzanne Bertera ${ }^{1}$, Christina Bagia ${ }^{2}$, Massimo Trucco $^{1}$, Wilson S. Meng2, ${ }^{2}$ and Yong Fan ${ }^{1, *}$

\section{Affiliations:}

1. Institute of Cellular Therapeutics, Allegheny Health Network, Pittsburgh, Pennsylvania, USA.

2. Division of Pharmaceutical Sciences, Mylan School of Pharmacy, Duquesne University, Pittsburgh, Pennsylvania, USA.

Running title: Bioengineering mini thymus with EAK16-II/EAKIIH6 hydrogel

Key words: $\quad$ Thymic epithelial cells, self-assembly, EAK16-II, hydrogel, thymus, thymopoiesis

* To whom correspondence should be addressed:

Yong Fan, $\mathrm{PhD}$

Institute of Cellular Therapeutics

Allegheny Health Network

1107 South Tower

320 East North Avenue

Pittsburgh, PA 15212-4772

Email: YFan@wpahs.org

Phone: 412-359-6382

Fax: $\quad 412-359-6987$

Or

Wilson Meng, PhD

Division of Pharmaceutical Sciences, Mylan School of

Pharmacy, Duquesne University, Pittsburgh, Pennsylvania, USA.

Email: Meng@duq.edu. 


\begin{abstract}
Herein, we highlight the technical feasibility of generating a functional mini thymus with a novel hydrogel system, based on a peptide-based self-assembly platform that can induce the formation of 3-D thymic epithelial cell (TEC) clusters. Amphiphilic peptide EAK16-II co-assembled with its histidinylated analogue EAKIIH6 into beta-sheet fibrils. When adaptor complexes (recombinant protein A/G molecules loaded with both anti-His and anti-EpCAM IgGs) were added to the mix, TECs were tethered to the hydrogel and formed 3-D mini clusters. TECs bound to the hydrogel composites retained their molecular properties; and when transplanted into athymic nude mice, they supported the development of functional T-cells. These mini thymic units of TECs can be useful in clinical applications to reconstitute T-cell adaptive immunity.
\end{abstract}




\section{Introduction}

Thymus is an essential gland for adaptive immunity. It continuously generates new T-lymphocytes to maintain a diverse repertoire of T-cell receptors (TCRs) that can effectively respond to any invading pathogens, while remaining unresponsiveness to self. Defective thymus function leads to immunodeficiency, malignancy, autoimmunity and other immunoregulatory disorders[1,2].

Successful T-cell development in the thymus depends on proper lympho-stromal interaction[3]. The stroma of a thymus is comprised primarily of a network of thymic epithelial cells (TECs), which can be grouped into two subsets: the cortical TECs (cTECs) and the medullary TECs (mTECs), based on their geometrical location and phenotypic markers. Bone marrow (BM)-derived lymphocyte precursors once entered into the thymus at the cortico-medullary junction region, first undergo lineage determination and positive selection in the cortical region. Subsequently, they migrate to the medullary region for negative selection of self-reactive thymocytes. Unlike other epithelial cells in the body, TECs are organized in a unique three-dimensional (3-D) fashion that is essential for their function and survival. TECs cultured as 2-D monolayers display altered patterns of expression of key genes for TEC function and growth, such as MHC II, FoxN1 and Tbata, and lose their capability to support T-cell development [4]. While tissue fragments isolated from murine fetal thymus can be cultured in vitro as reaggregated fetal thymus organ culture (RFTOC), the protocol does not work well with TECs from postnatal mice, presumably due to lack of TEC progenitors [5]. While numerous attempts have been made to mimic the 3-D ECM environment to support TEC culture, the success has been limited to date. In these approaches, isolated TECs were seeded into scaffolds made of artificial 
biomaterials or chambers coated with collagens, either alone or in conjunction with other stromal cells[6, 7]. Among the many factors (e.g. the composition of the extracellular matrix proteins, the presence of supporting stromal cells, and the growth factors supplemented) that could affect the function of the TECs in vitro, one potential issue of this "bottom-up" approach is the lack of geometrical organization of the TECs in the artificial scaffolds, which is presumably important for establishing proper TEC-TEC and TEC-thymocyte interaction for successful thymopoiesis.

The environmentally-responsive, $\beta$-fibril forming EAK series of low molecular weight peptides are versatile building blocks for making 3-D, tissue engineering scaffolds[8]. Upon exposure to an environment of high ionic strength, the amphiphilic peptide EAK16-II (AEAEKAKAEAEAKAK) undergoes liquid to gel transition [9] and coassembles with its C-terminal histidinylated analogue EAKII-H6 (AEAEKAKAEAEAKAKHHHHHH)[8, 10-12]. Integrating the histidine (His)-tags in the hydrogel complexes provides a docking mechanism, transforming the self-assembled hydrogel into a platform that can be used to deliver protein drugs and other biomolecules. We have previously shown that fluorescent dye-conjugated antibodies can be anchored to the hydrogel using a linker complex comprised of anti-His antibodies ( $\alpha \mathrm{H} 6 \operatorname{IgG})$ and the Fcbinding, recombinant protein $A / G$ (rpAG)[11]. When injected into the mice, the antibodies remained localized for at least 13 days [12]. EAK16-II/EAKIIH6 composites displaying antiCD4 IgGs through the $\alpha \mathrm{H} 6 / \mathrm{rpAG}$ linker supported on polyethylene terephthalate (PET) membranes could capture T-lymphocytes as clusters when a mixed population of mouse lymphocytes, including T, B and NK cells, were passed through[11]. These studies suggest that the EAK16-II/EAKIIH6 composite can be utilized to induce the 3-D aggregation of TECs 
for in vitro culture. Here, we showed that EAK16-II/EAKIIH6 composites displaying antiEpCAM IgGs promoted the formation of 3-D mini clusters of TECs that can function as mini thymus units in vivo to support T-cell development.

\section{Materials and Methods}

\section{1. $\quad$ Mice}

All animal protocols were reviewed and approved by the Institutional Animal Care and Use Committee of the Allegheny Health Network (AHN)/Allegheny Singer Research Institute (ASRI). Mice were housed in a specific pathogen free environment. B6 (C57BL/6J) mice were purchased from the Jackson Laboratory (Bar Harbor, ME); athymic B6.nude mice were obtained either from the Jackson Laboratory or from Taconic (Germantown, NY); similar results were obtained from both nude mouse strains.

\subsection{Viscosity measurement}

Samples of EAK16-II with or without EAKIIH6 were prepared in phosphate buffered saline pH 7.4 (PBS) and drew into positive displacement pipettes (RheoSense Inc., San Ramon, CA 94583). Specifically, EAK16-II was prepared in different concentrations (0.01 $2 \mathrm{mg} / \mathrm{mL}$ ) in two solvents (PBS and distilled water). The EAK16-II with EAKIIH6 in PBS was prepared at the ratio $4: 1$ and the final concentrations were $1.5 \mathrm{mg} / \mathrm{mL}$ and 0.56 $\mathrm{mg} / \mathrm{mL}$ respectively. The stock solutions of EAK16-II and EAKIIH6 were prepared in distilled water at the level of $10 \mathrm{mg} / \mathrm{mL}$ and $7.5 \mathrm{mg} / \mathrm{mL}$ respectively. Measurements were performed using the $\mu$ VISC unit (RheoSense Inc.) equipped with a temperature control module set at $25^{\circ} \mathrm{C}$. Samples were subjected to different shear rates by changing the flow rate through the capillaries (depth $=50 \mu \mathrm{m}$ ) in the sensor cartridge (HA01-01). The 
instrument is capable of measuring viscosity between 0.2 to $100 \mathrm{mPa}^{*} \mathrm{~s}$ up to $2 \%$ accuracy in a range shear rates 6.5 to $5,850 \mathrm{~s}^{-1}$.

\subsection{Isolation of thymic epithelial cells (TECs)}

Thymi were harvested from 3-4 week old B6 mice, and TECs were isolated as previously described with modification[13]. In brief, thymus glands $(n=3-4)$ were needle dissected into small pieces of approximately $1 \mathrm{~mm}^{3}$, and digested with the Liberase TM solution $[0.025 \mathrm{mg} / \mathrm{ml}$ Liberase TM (Roche Applied Science, Indianapolis), 0.2mg/ml DNase I (Roche Applied Science), and 10mM HEPES in RPMI-1640 (Life Technologies, Carlsbad, $\mathrm{CA})]$ at $37^{\circ} \mathrm{C}$ for a total of 18 minutes ( 3 rounds of 6 minutes each). All fractions were pooled, and slowly layered on top of 20\% OptiPrep Cell Separation Media (Thermo Scientific, Rockford, IL). They were incubated with magnetic bead-conjugated anti-CD45 antibodies, and subjected to negative selection of CD45- thymic stromal cells (e.g. TECs and thymic fibroblasts) with MACS separation technology (Miltenyi Biotec, Auburn, CA). Approximately 0.5-1 million TECs were routinely obtained per thymus.

2.4. Construction of TEC/EAK hydrogel mini thymus units

EAK16-II (AcNH-AEAEAKAKAEAEAKAK-CONH 2$)$ and EAKIIH6 (AcNHAEAEAKAKAEAEAKAKHHHHHH-CONH 2 ) peptides were custom synthesized by American Peptide Company (Sunnyvale, CA) at $>95 \%$ purity. Both the EAK16-II and the EAKIIH6 peptides were reconstituted in sterile water at $10 \mathrm{mg} / \mathrm{mL}$ and $7.5 \mathrm{mg} / \mathrm{mL}$, respectively. Recombinant protein A/G (rpAG, Pierce Biotechnology, Rockford, IL) was reconstituted with sterile deionized water at a concentration of $0.25 \mathrm{mg} / \mathrm{mL}$. Rabbit anti-His-tag polyclonal antibody ( $\alpha \mathrm{H} 6)$ was obtained from AnaSpec, Inc $(1 \mathrm{mg} / \mathrm{mL}$, San Jose, CA). Purified rat anti-mouse EpCAM antibody (clone G8.8) was purchased from Biolegend (0.5 
$\mathrm{mg} / \mathrm{mL}$, San Diego, CA). To construct the TEC/EAK mini thymus units, 150,000 TECs were stained with the tri-component adaptor complexes (preassembled with $4 \mu \mathrm{g}$ of $\alpha \mathrm{H} 6,4 \mu \mathrm{g}$ of aEpCAM IgG and $0.65 \mu \mathrm{g}$ of rpAG; molar ratio, 2:2:1), and resuspended in $10 \%$ sucrose (Sigma) after extensive wash with $10 \%$ sucrose. The self-assembling peptide solution was prepared by mixing EAK16-II and EAKIIH6 peptides at 4:1 ratio. $\alpha$ EpCAM adaptor labeled TECs and the EAK peptide mixture were mixed at 1:1 ratio (volume) and incubated at room temperature for 5 minutes. To initiate the gelafication, complete RPMI-10 medium was added to the TEC/EAK hydrogel mixture.

\subsection{Detection of live TECs in the EAK16-II/EAKIIH6 hydrogel}

LIVE/DEAD viability kit for mammalian cells (Life technologies, Grand Island, NY) was used to detect live cells in TEC clusters embedded in the hydrogel in vitro, following the fluorescence microscopy protocol suggested by the manufacturer. The kit discriminates live from dead cells by simultaneously staining with green-fluorescent calcein-AM, an indicator of intracellular esterase activity, and red-fluorescent ethidium homodimer-1 (EthD-1), an indicator of loss of plasma membrane integrity. Briefly, the TEC/EAK hydrogel composites were washed three times with Dulbecco's phosphate-buffered saline (D-PBS). Staining solution was prepared by diluting stock solutions of both EthD-1 and calcein-AM in D-PBS to a final concentration of $4 \mathrm{uM}$ and $2 \mathrm{uM}$, respectively. The TEC/EAK composites were incubated in the staining solution for 30 minutes at room temperature, and examined under the inverted laser scanning fluorescence microscope (FV1000, Olympus).

\subsection{T-cell proliferation assay}

T-cells were isolated from the spleens and/or lymph nodes of B6.nude mice $(n=5)$ that were transplanted with the TEC/EAK hydrogel mini thymus grafts (16-weeks post op). 
They were first labeled with carboxyfluorescein diacetate, succinimidyl ester (CFSE), and subsequently stimulated with allogeneic antigen presenting cells (harvested from the spleens of $\mathrm{CBA} / \mathrm{J}$ mice). Proliferation of T-cells was evaluated by the dilution of CFSE intensities with FCM analyses, as previously described. Briefly, T-cells were suspended at a

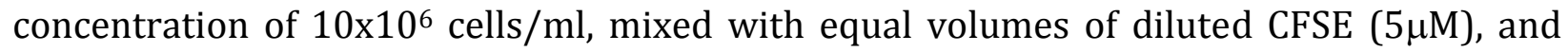
incubated at $37^{\circ} \mathrm{C}$ for $10 \mathrm{~min}$. After extensive wash, $100 \mu$ of CFSE-labeled in RMPI-10 $\left(2.5 \times 10^{6}\right.$ cells $\left./ \mathrm{ml}\right)$ were mixed with $2.5 \times 10^{5} \mathrm{~T}$-cell depleted, mitomycin C (Sigma) treated splenocytes harvested from CBA/J mice, and cultured for 7 days in wells of 96-well plates. Cells were stained with LIVE/DEAD fixable violet dyes (Life Technologies), followed by labeling with anti-CD4 and -CD8 antibodies (BD Biosciences) for FCM analyses. Unless specified otherwise, all the experiments were run in triplicate and repeated at least three times.

\subsection{RNA analysis}

Total RNA was extracted from freshly isolated 150,000 TECs, or TEC/EAK mini thymus units cultured in vitro for 14 days with RNeasy Micro Plus kit, following the manufacturer's protocol (Qiagen). The RNA samples were eluted in $30 \mu \mathrm{L}$ of $10 \mathrm{mM}$ Tris. $\mathrm{HCl}$ (PH7.5) buffer with 1mM of ethylenediaminetetraacetic acid (EDTA). $8 \mu \mathrm{L}$ of RNA samples were reverse-transcribed into cDNAs with Superscript III cDNA kit, with random hexamers as primers in a $20 \mu \mathrm{L}$ RT reaction (Invitrogen). $2 \mu \mathrm{L}$ cDNA samples (1:10 dilution) were used as templates for PCR amplification of the gene of interest. Sequences of primer pairs are listed below:

FoxN1 (F) 5'-TGACGGAGCACTTCCCTTAC-3', (R) 5'-GACAGGTTATGGCGAACAGAA3'; EpCAM (F) 5'-AGAATACTGTCATTTGCTCCAAACT-3', (R) 5'- 
GTTCTGGATCGCCCCTTC-3'; CCL25 (F) 5'-GAGTGCCACCCTAGGTCATC-3', (R) 5'CCAGCTGGTGCTTACTCTGA-3'; Aire (F) 5'-AgCACCTTCCTCTTGGAAAC-3', (R) 5'CCCACTTTCTGCTCATCTCTA-3'; Krt5 (F) 5'-CAGGGCACCAAGACCATAAA-3'; (R) 5'CTGTTGCAGCTCCTCATACTT-3'; Krt8 (F) 5'-CGTCTGTGGTGCTGTCTATG-3', (R) 5'CTTGGTCTGGGCATCCTTAAT-3'; Krt14 (F) 5'-GGATGCTGAGGAATGGTTCT-3', (R) 5'CTTTGGTCTCCTCCAGGTTATT-3'; 18S (F) 5'-AAACGGCTACCACATCCAAG-3', (R) 5'CCTCCAATGGATCCTCGTTA-3'. All the PCR reactions, except for 18S, were performed at $60^{\circ} \mathrm{C}$ annealing temperature for 40 cycles. To examine the expression of $18 \mathrm{~S}$, cDNA samples were diluted 1000 times and amplified for 28 cycles.

\subsection{Flow Cytometry.}

Flow cytometric analysis was performed on the BD FACSCalibur flow cytometer (BD Biosciences, San Jose, CA) and analyzed with the CellQuest Pro software (BD Biosciences) or Flowjo. Splenocytes were treated with red blood cell lysis buffer (Sigma-Aldrich, St. Louis, MO). Before staining with antibodies of specific markers, single cells (isolated from either the spleens or the lymph nodes) were labeled with anti-CD16/32 IgGs for Fcreceptor blocking. The following antibodies were purchased from BD Biosciences: antiCD16/32 (2.4G2), anti-CD4 (H129.9), anti-CD45 (30-F11), anti-CD3 (145-2C11), antiEpcam (g8.8), anti-CD62L (MEL-14), and anti-CD69 (H1.2F3). Staining buffer: phosphate buffered saline (PBS, calcium and magnesium free, Invitrogen) supplemented with $1 \%$ bovine serum albumin (BSA, Sigma-Aldrich) and $0.1 \%$ sodium azide (Sigma-Aldrich). Intracellular staining of the Foxp3 protein was performed with commercial kit purchased from eBiosciences (San Diego, CA), following manufacturer's suggested protocol. 


\section{Results and Discussion}

\subsection{EAK16-II/EAKIIH6 mediated the formation of mini-thymus complex in vitro}

To investigate whether we can utilize the co-assembly properties of the EAK16-II /EAKIIH6 system to promote the 3-D aggregation of TECs, we generated the heptamolecule adaptor complexes to anchor TECs to the hydrogel matrix. The adaptor complex was comprised of one recombinant protein $A / G(r p A G)$ molecule that contains six Fcbinding sites, three anti-His IgG molecules and three anti-Epcam IgG molecules (Figure 1A). The high-affinity interaction between the His-Tag on the EAKIIH6 and the anti-His IgG served as a molecular bridge to anchor the Epcam-expressing TECs on the EAK16II/EAKIIH6 structure. With similar adaptor design, we have previously shown that the EAK16-II/EAKIIH6 hydrogel matrix can successfully retain fluorescent dye-conjugated IgG molecules for up to 13-days in vitro. Moreover, polycarbonate mesh filters coated with antiCD4 IgG-loaded EAK16-II/EAKIIH6 composites could capture CD4+ T-cells as clusters when a mixed population of lymphocytes (including T, B and natural killer cells) passed through.

In the current study, viscosity the self-assembling peptide EAK16-II that forms the material network was measured using capillary rheometry[14]. This method determines viscosity by measuring pressure difference as sample thrusts through microfluidic channels, without an air-liquid interface as a potential confounding factor. As expected, EAK16-II exhibits concentration- dependent viscosities, with $1.5 \mathrm{mg} / \mathrm{ml}$ detected as the inflection point at low shear rates (Figure 1B, upper panel). Viscosities at all concentrations were found below $2.5 \mathrm{mPa}^{*}$ s measured at shear rates below $8,900 \mathrm{~s}^{-1}$, the 
apparent shear rate estimated for samples expulsed through a 27 Gauge needle using a 1-cc syringe [Zarraga, 2013]. Addition of EAKHII6 to EAK16-II did not raise viscosity above 2.5 $\mathrm{mPa}$, even at a low shear rate of $1600 \mathrm{~s}^{-1}$ (Figure 1B, lower panel). These data show that the hydrogels mixture can be injected through conventional syringe injection.

To induce the formation of TEC clusters, TECs enriched by magnetic-activated sorting were labeled with the adaptor complexes and mixed with EAK16-II/EAKIIH6 solution under low ion conditions. Self-assembly of EAK16-II/EAKIIH6 hydrogel was promoted by supplementing the mixture with culture medium. As shown in Figure 1C, TECs formed clusters of 20-30 cells in the hydrogel matrix, suggesting that the EAK16II/EAKIIH6 hydrogel system could induce the aggregation of TECs.

Since the 3-D thymic stromal configuration is essential to the survival of TECs, we next examined the viability of TECs embedded in the EAK16-II/EAKIIH6 hydrogel with Calcein-AM and ethidium homodimer-1 (EthD-1) staining. In this assay, the presence of green calcein-AM signal indicates live cells with intracellular esterase activity, whereas redfluorescent EthD-1 is retained in dead cells with compromised plasma membrane integrity. As shown in Figure 1D, most of the TECs remained alive after 3-days of culture within the hydrogel. To further demonstrate that the EAK16-II/EAKIIH6 matrix can prolong the survival of the anchored TEC clusters, we extended the culture to 2-weeks and measured the levels of 18S RNA transcription with quantitative RT-PCR, as an indicator of cell viability. As control, TECs were cultured in hydrogel matrix supplemented with adaptor complexes made of isotype IgGs, instead of anti-EpCAM IgGs. In comparison to controls, a significantly higher level of $18 \mathrm{~S}$ RNA transcription was observed, suggesting that the 
EpCAM IgG adaptor-mediated 3-D configuration of TEC clusters can indeed prolong the survival of TECs (Figure 1E).

To investigate whether TEC clusters anchored to the EAK16-II/EAKIIH6 hydrogel matrix can maintain their molecular properties, we performed RT-PCR analysis of genes that were expressed in the thymus stroma (Figure 1F). Even after two weeks of in vitro culture, we were able to detect the expression of genes specific to thymic epithelium (EpCAM), as well as those of different TEC subsets (e.g. Krt5 and Krt14 for mTECs, and Krt8 for cTECs) $[15,16]$. Transcriptions of TEC-specific transcription factors (e.g. FoxN1 and Tbata) were also detected $[17,18]$. In addition, expression of signaling molecules (e.g. CCL25) important for lympho-stromal interactions to support T-lymphopoiesis was also maintained (Figure 1G). Transcription of the Aire gene, which is expressed exclusively in mTECs to regulate the ectopic expression of a broad range of tissue specific antigens in the thymus for negative selection of autoreactive thymocytes, was also detected[19]. These results suggest that TECs embedded in the EAK16-II/EAKIIH6 as clusters not only can maintain their thymic epithelium identity in vitro, but might also be able to retain their functionalities to support T-cell development.

\subsection{The TEC/EAK Mini thymus units support T-lymphopoiesis in vivo}

We have previously shown that when subcutaneously injected into BALB/c mice, fluorescent dye-labeled IgGs anchored to the EAK16-II/EAKIIH6 hydrogel with the rpA/G adaptor complex remained stable for more than 2-weeks[12], suggesting its potential application in vivo. To examine whether the EAK16-II/EAKIIH6 based mini-thymus units can support the development of T-cells in vivo, we transplanted them underneath the kidney capsules of athymic B6.FoxN1\% nude mice (B6.nude). Flow cytometry (FCM) 
analyses revealed the presence of circulating CD45+CD3+ T-cells as early as 5-weeks post operation $(\sim 2-4 \%$, Figure $2 A)$, in striking contrast to the background levels of CD45+CD3+ cells found in the blood of untreated nude mice $(0.164 \pm 0.017 \%, n=6)$ at $8-16$ weeks of age. To further examine the development of T-cells in the B6.nude mice transplanted with the mini-thymus grafts, we examined the composition of T-cells in secondary lymphoid organs. Both CD3+CD4+ and CD3+CD8+ T-cells are present (Figure 2B), suggesting that the mini thymus made of TEC/EAK composites can support thymopoiesis. While the numbers of CD4+ T-cells were significantly less than those of the wild type B6 controls, comparable numbers of CD8+ T-cells were found in the spleens and lymph nodes of the thymus reconstructed nude mice (Figure 2C). Most of the CD8+ cells displayed the CD62 $\mathrm{L}^{\text {high }}$ CD69low phenotype of naïve T-cells. In contrast, majorities of the CD4+ T-cells exhibited the CD62L $\mathrm{L}^{\text {low }} \mathrm{CD} 69^{\text {high }}$ phenotype of activated T-cells (Figure 2D). These results suggest that there is a preference of CD8+ SP thymocyte development in the mini thymus made of TEC/EAK composites.

To examine the functionality of the mature T-cells in the thymus reconstructed B6.nude mice, we performed mixed leukocyte reaction (MLR) assay to examine their proliferating response upon TCR stimulation. Both CD4+ and CD8+ T-cells displayed drastic proliferation responses similar to those of wild-type B6 mouse, indicating that these T-cells were capable to react to alloantigens (Figure 2E). In contrast, only limited, background levels of proliferation were observed when syngeneic antigen presenting cells (APCs) were supplemented, suggesting that mature T-cells derived from the TEC/EAK mini thymus units are self-tolerant. 
Taken together, we showed that TECs anchored to EAK16-II/EAKIIH6 hydrogel with anti-EpCAM IgG containing tri-component adaptor complex could self-assemble into 3-D aggregates. This 3-D configuration could retain, to a certain degree, the properties of the TECs in vitro, and enable them to function as mini thymus units to support the development of mature T-cells when engrafted into athymic nude mice. Of note, a preferential development of CD8+ over CD4+ T-cells was observed. While the underlying mechanism of CD4/CD8 lineage commitment during thymopoiesis remains under intensive debate, current evidence suggests that both the duration and intensity of TCR signaling are important for lineage determination[20]. Deficient CD4+ T-cell development in the TEC/EAK mini thymus units might reflect inadequate interaction between the CD4+CD8+ double positive (DP) thymocytes and the MHC II gene expressing TECs[21]. One possible approach to extend the interactions between DP thymocytes and the TECs is to increase the density of the mini thymus units in the EAK hydrogel. Prolongation of the interactions between thymocytes and self-antigen expressing mTECs might also promote the development of CD4+Foxp3+ natural regulatory T-cells (nTreg cells), as it has been previously shown that the MTEC compartment is essential for the lineage-determination and survival of nTreg cells[22-25]. In addition, close contacts between T-cell progenitors and subsets of TECs could also facilitate the development of $\gamma \delta$ T-cells, the minor population of T-cells that are highly enriched at epithelial surfaces and exhibit various activities of immune regulation and immunosurvelliance [26,27]. This can be achieved by optimizing the conditions to generate the TEC/EAK mini thymus units, such as increasing the concentration of the TECs, optimizing the ratio of EAK16-II/EAKIIH6, or including other types of thymic stromal cells into the composites. 
Another caveat in the current form of the TEC/EAK composite is that there is no segregation of cTECs and mTECs, whereas normal thymus is comprised of cortical and medullary regions with distinctive functions. One way to overcome this shortcoming is to design adaptor complexes with antibodies specifically targeting either cTECs (e.g. anti-Krt8 or -Krt18 IgGs), or mTECs (e.g. anti-Krt5 or -Krt14 IgGs). Bridging the TECs to the EAK16II/EAKIIH6 hydrogel with a mixture of cTEC- and mTEC-specific adaptor complexes, in place of the ones with anti-EpCAM IgGs, will promote the formation of TEC aggregates of specific subsets that serve distinctive roles in T-cell development.

Nevertheless, the tri-component adaptor complex and self-assembling EAK16II/EAKIIH6 system provide a versatile tool to organize the TECs in the 3-D configuration, mimicking those of the thymus stroma. With further optimization, the TEC/EAK system might be used to generate injectable mini thymus units to treat immunomodulatory disorders by either modifying $\mathrm{T}$ cell adaptive immunity at central levels or promoting the development of specific T-cell subsets (e.g. nTregs) to modulate immune homeostasis. 


\section{Acknowledgements}

We thank Robert Lakomy and Alex Syche for their exemplary technical assistance. We also thank Dr. Nick Giannoukakis for insightful discussions and suggestions. Funding: This study was supported by Department of Defense grant \# W81XWH-10-1-1055 (MT) and by NIH grant R21-AI113000 (WSM). The authors declare no competing financial interests. 


\section{References}

[1] Y. Fan, W.A. Rudert, M. Grupillo, J. He, G. Sisino, M. Trucco, Thymus-specific deletion of insulin induces autoimmune diabetes, The EMBO Journal, 28 (2009) 2812-2824.

[2] H. von Boehmer, The thymus in immunity and in malignancy, Cancer immunology research, 2 (2014) 592-597.

[3] Y. Takahama, Journey through the thymus: stromal guides for T-cell development and selection, Nat Rev Immunol, 6 (2006) 127-135.

[4] K.L. Anderson, N.C. Moore, D.E. McLoughlin, E.J. Jenkinson, J.J. Owen, Studies on thymic epithelial cells in vitro, Developmental and comparative immunology, 22 (1998) 367-377.

[5] N. Seach, M. Hammett, A. Chidgey, Isolation, characterization, and reaggregate culture of thymic epithelial cells, Methods in molecular biology (Clifton, N.J.), 945 (2013) 251-272.

[6] N. Seach, M. Mattesich, K. Abberton, K. Matsuda, D.J. Tilkorn, J. Rophael, R.L. Boyd, W.A. Morrison, Vascularized tissue engineering mouse chamber model supports thymopoiesis of ectopic thymus tissue grafts, Tissue engineering. Part C, Methods, 16 (2010) 543-551.

[7] S. Pinto, K. Schmidt, S. Egle, H.J. Stark, P. Boukamp, B. Kyewski, An organotypic coculture model supporting proliferation and differentiation of medullary thymic epithelial cells and promiscuous gene expression, J Immunol, 190 (2013) 1085-1093.

[8] Y. Wen, H.R. Kolonich, K.M. Kruszewski, N. Giannoukakis, E.S. Gawalt, W.S. Meng, Retaining antibodies in tumors with a self-assembling injectable system, Mol Pharm, 10 (2013) 1035-1044.

[9] S. Zhang, T. Holmes, C. Lockshin, A. Rich, Spontaneous assembly of a selfcomplementary oligopeptide to form a stable macroscopic membrane, Proc Natl Acad Sci U S A, 90 (1993) 3334-3338.

[10] Y. Wen, W. Liu, C. Bagia, S. Zhang, M. Bai, J.M. Janjic, N. Giannoukakis, E.S. Gawalt, W.S. Meng, Antibody-functionalized peptidic membranes for neutralization of allogeneic skin antigen-presenting cells, Acta Biomater, 10 (2014) 4759-4767.

[11] Y. Zheng, Y. Wen, A.M. George, A.M. Steinbach, B.E. Phillips, N. Giannoukakis, E.S. Gawalt, W.S. Meng, A peptide-based material platform for displaying antibodies to engage $\mathrm{T}$ cells, Biomaterials, 32 (2011) 249-257.

[12] Y. Wen, S.L. Roudebush, G.A. Buckholtz, T.R. Goehring, N. Giannoukakis, E.S. Gawalt, W.S. Meng, Coassembly of amphiphilic peptide EAK16-II with histidinylated analogues and implications for functionalization of beta-sheet fibrils in vivo, Biomaterials, 35 (2014) 5196-5205.

[13] Y. Fan, G. Gualtierotti, A. Tajima, M. Grupillo, A. Coppola, J. He, S. Bertera, G. Owens, M. Pietropaolo, W.A. Rudert, M. Trucco, Compromised central tolerance of ICA69 induces multiple organ autoimmunity, Journal of Autoimmunity, (2014).

[14] I.E. Zarraga, R. Taing, J. Zarzar, J. Luoma, J. Hsiung, A. Patel, F.J. Lim, High shear rheology and anisotropy in concentrated solutions of monoclonal antibodies, Journal of pharmaceutical sciences, 102 (2013) 2538-2549.

[15] B. Li, J. Li, B.H. Devlin, M.L. Markert, Thymic microenvironment reconstitution after postnatal human thymus transplantation, Clinical Immunology, 140 (2011) 244-259.

[16] E.N. Lee, J.K. Park, J.R. Lee, S.O. Oh, S.Y. Baek, B.S. Kim, S. Yoon, Characterization of the expression of cytokeratins 5,8 , and 14 in mouse thymic epithelial cells during thymus regeneration following acute thymic involution, Anat Cell Biol, 44 (2011) 14-24. 
[17] C.S. Nowell, N. Bredenkamp, S. Tetelin, X. Jin, C. Tischner, H. Vaidya, J.M. Sheridan, F.H. Stenhouse, R. Heussen, A.J.H. Smith, C.C. Blackburn, Foxn1 Regulates Lineage Progression in Cortical and Medullary Thymic Epithelial Cells But Is Dispensable for Medullary Sublineage Divergence, Plos Genetics, 7 (2011).

[18] G. Anderson, S. Baik, J.E. Cowan, A.M. Holland, N.I. McCarthy, K. Nakamura, S.M. Parnell, A.J. White, P.J. Lane, E.J. Jenkinson, W.E. Jenkinson, Mechanisms of Thymus Medulla Development and Function, Curr Top Microbiol Immunol, (2013).

[19] M.S. Anderson, Projection of an Immunological Self Shadow Within the Thymus by the Aire Protein, Science, 298 (2002) 1395-1401.

[20] X. He, K. Park, D.J. Kappes, The role of ThPOK in control of CD4/CD8 lineage commitment, Annual review of immunology, 28 (2010) 295-320.

[21] A.C. Carpenter, R. Bosselut, Decision checkpoints in the thymus, Nat Immunol, 11 (2010) 666-673.

[22] J.M. Coquet, J.C. Ribot, N. Babala, S. Middendorp, G. van der Horst, Y. Xiao, J.F. Neves, D. Fonseca-Pereira, H. Jacobs, D.J. Pennington, B. Silva-Santos, J. Borst, Epithelial and dendritic cells in the thymic medulla promote CD4+Foxp3+ regulatory $\mathrm{T}$ cell development via the CD27-CD70 pathway, The Journal of experimental medicine, 210 (2013) 715-728.

[23] D. Nazzal, A. Gradolatto, F. Truffault, J. Bismuth, S. Berrih-Aknin, Human thymus medullary epithelial cells promote regulatory T-cell generation by stimulating interleukin2 production via ICOS ligand, Cell death \& disease, 5 (2014) e1420.

[24] K. Aschenbrenner, L.M. D'Cruz, E.H. Vollmann, M. Hinterberger, J. Emmerich, L.K. Swee, A. Rolink, L. Klein, Selection of Foxp3+ regulatory T cells specific for self antigen expressed and presented by Aire+ medullary thymic epithelial cells, Nature immunology, 8 (2007) 351-358.

[25] J.E. Cowan, S.M. Parnell, K. Nakamura, J.H. Caamano, P.J. Lane, E.J. Jenkinson, W.E. Jenkinson, G. Anderson, The thymic medulla is required for Foxp3+ regulatory but not conventional CD4+ thymocyte development, The Journal of experimental medicine, 210 (2013) 675-681.

[26] G. Lee, K.Y. Kim, C.H. Chang, M.G. Kim, Thymic epithelial requirement for gammadelta T cell development revealed in the cell ablation transgenic system with TSCOT promoter, Molecules and cells, 34 (2012) 481-493.

[27] M. Girardi, Immunosurveillance and immunoregulation by gammadelta $\mathrm{T}$ cells, The Journal of investigative dermatology, 126 (2006) 25-31. 


\section{Figure Legends}

Figure 1. Generation of the TEC/EAK mini thymus complex in vitro. A. Schematic drawing showing the strategies to generate the TEC/EAK mini thymus complex. The tricomponent adaptor complex was formed by mixing anti-EpCAM antibody (Ab), anti-His Ab and recombinant protein $\mathrm{A} / \mathrm{G}(\mathrm{pA} / \mathrm{G}$, which contains six Fc-binding sites) at 3:3:1 molar ratio. Isolated thymic epithelial cells (TECs) were stained with the adaptor complexes, and mixed with EAK16-II (EAK) and EAKIIH6 (EAKH6) mixture in 10\% sucrose. Gelafication is initiated by adding complete RMPI-10 medium to the TEC/EAK mixture. B. Upper panel: concentration dependence of EAK16-II viscosity in buffered saline measured at different shear rates; lower panel: viscosity of mixtures of EAK16-II (1.5 mg/ml) and EAKIIH6 (0.56 $\mathrm{mg} / \mathrm{ml}$ ) dissolved in $96 \mathrm{mM} \mathrm{NaCl}$ at $1600 \mathrm{~s}^{-1}$ (points represent replicate measurements of samples prepared independently). C. Representative Phase contrast microscopic images (50x) of TEC aggregates (arrows) in EAK16-II/EAKH6 hydrogel. D. Representative fluorescent microscopic images of TEC aggregates in EAK16-II/EAKH6 hydrogel cultured in vitro for 3 days. Live cells were discriminated from the dead cells by their intracellular esterase activities to generate green fluorescent calcein-AM (green) and their capabilities to exclude the red-fluorescent ethidium homodimer-1 (EthD-1, red) from entering the nucleus. Left panel, low magnification image (100x) of TEC aggregates (white arrows); right panel, high magnification image (400x) of TEC aggregates. E. Real-time RT-qPCR analysis of 18S gene transcription. TECs were anchored to the EAK hydrogel with adaptor complexes containing either anti-EpCAM IgG (blue line, left panel) or isotype control IgG, and cultured for 14-days. Left panel, representative image of amplification curves: blue line, TEC/EAK hydrogel with anti-EpCAM IgG; red line, TEC/EAK hydrogel with isotype IgG; green line, 
negative control; Right panel, fold difference of $18 \mathrm{~S}$ transcription levels between TECs aggregates with anti-EpCAM IgG $(n=4$, open bar) and those with isotype $\operatorname{IgG}(n=4$, control). Data were presented as mean \pm sem. F. RT-PCR analyses of thymic stroma-specific gene transcription. The TEC/EAK mini thymus units were cultured in vitro for 2-weeks. TEC, freshly isolated TECs; Neg, negative control.

Figure 2. TEC/EAK mini thymus units support T-lymphopoiesis in vivo. A. Percentages of CD45+CD3+ T-cells in the circulating peripheral blood leukocytes in B6.nude mice $(n=5)$ transplanted with the TEC/EAK mini thymus units. B. FCM analyses of T-lymphocytes in B6.nude mice. Splenocytes and lymph node cells were stained for CD3, CD45, CD4 and CD8, and were gated on the CD45+CD3+ population. Representative FCM profiles for CD4+ and CD8+ T-cells in the spleens (SPL) and lymph nodes (LN) of TEC/EAK mini thymus reconstructed B6.nude mice $(n=5)$ and B6 controls $(n=3)$. C. The total numbers of CD4 and CD8 T-cells in the spleens and lymph nodes of thymus reconstructed B6.nude $(n=5)$ and B6 (n=3) mice. D. FCM analyses of activation marker expression in CD4 and CD8 T-cells. Tcells harvested from spleens (SP) and lymph nodes (LN) of B6 (n=3) and TEC/EAK thymus transplanted B6.nude (EAK, $\mathrm{n}=5$ ) mice were stained for CD62L and CD69. Numbers in the representative FCM profiles indicate the frequencies of cells within the indicated areas. E. Proliferation of T-cells in response to TCR stimulation. T-cells harvested from the spleens of the TEC/EAK mini thymus transplanted B6.nude $(n=3)$ were labeled with CFSE and cultured for 7 days in the presence or absence of T-cell depleted allogeneic antigen presenting cells (APCs) isolated from CBA/J mice. Cells were stained for CD3 and B220, gated on the CD3+B220-populations were analyzed by FCM for CFSE levels. Representative histograms of FCM results are shown. 
Figure 1

\section{A}

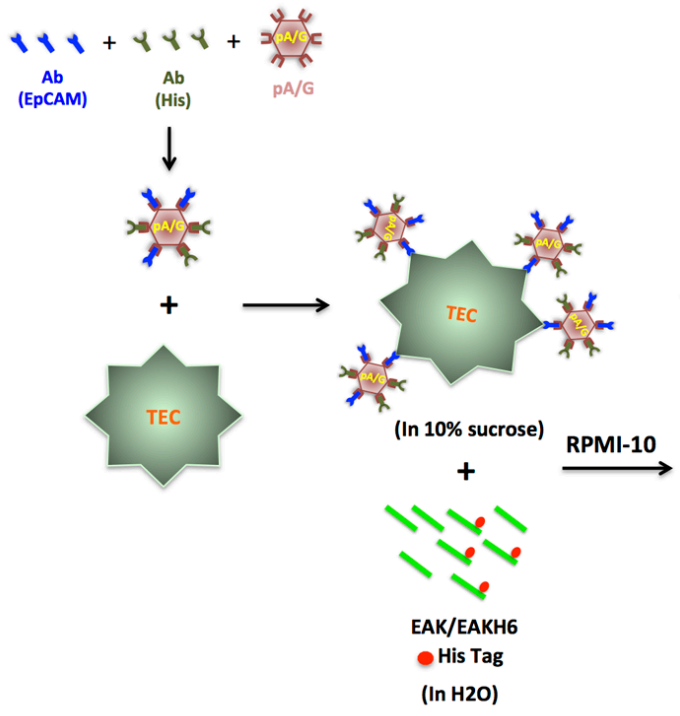

C
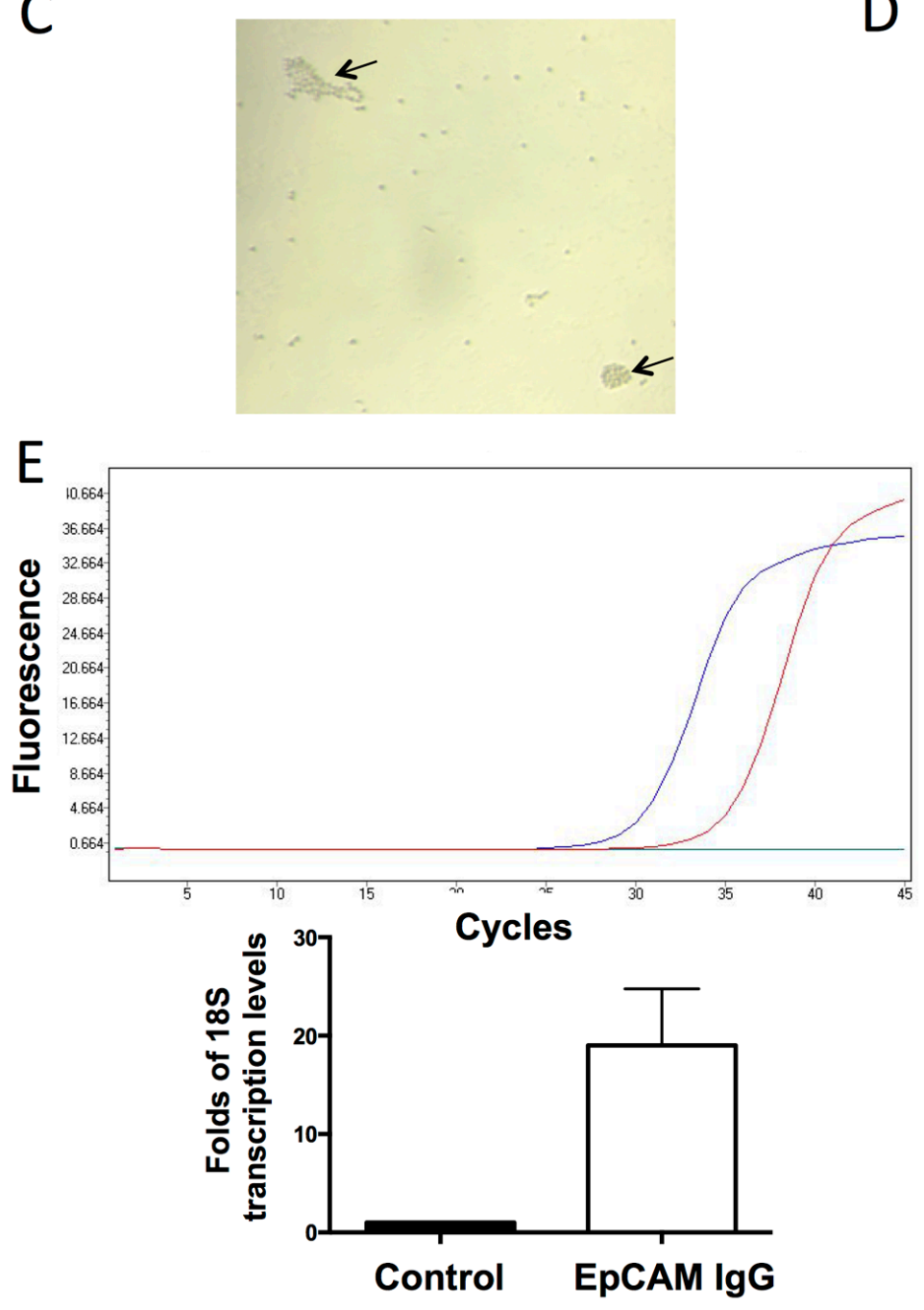

B
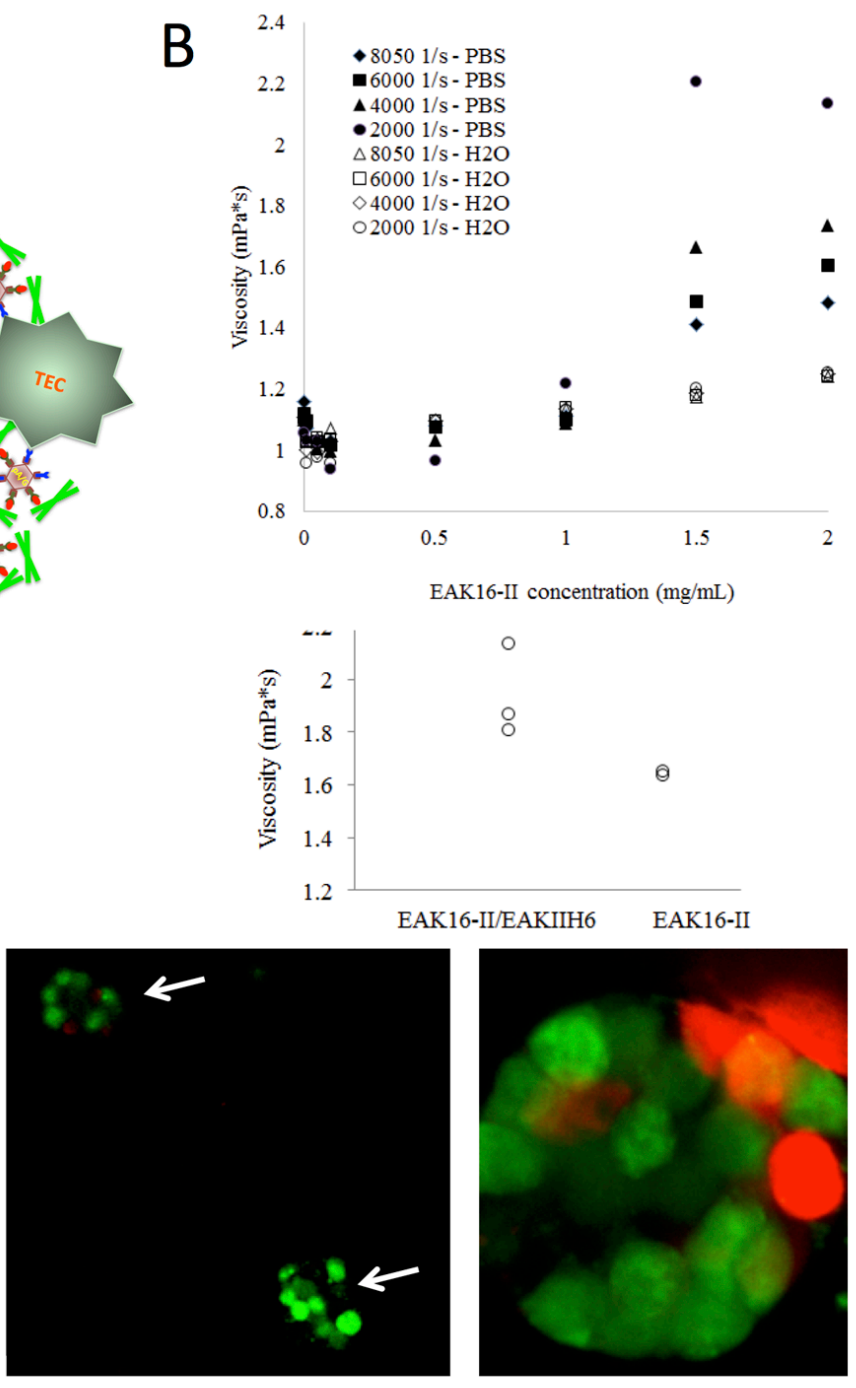

F
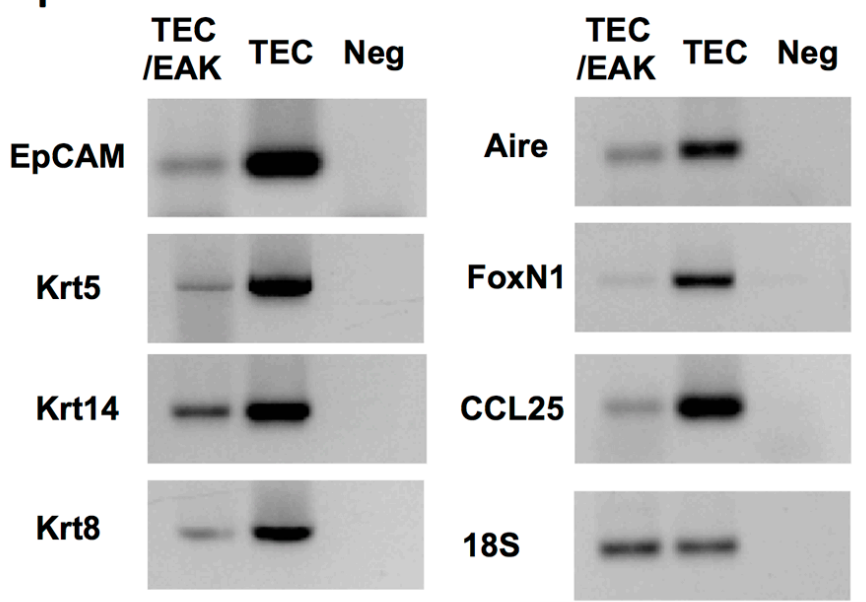
Figure 2

A

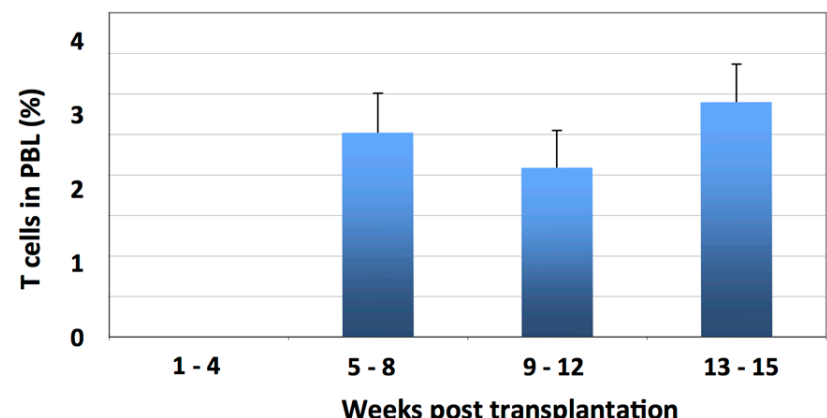

C
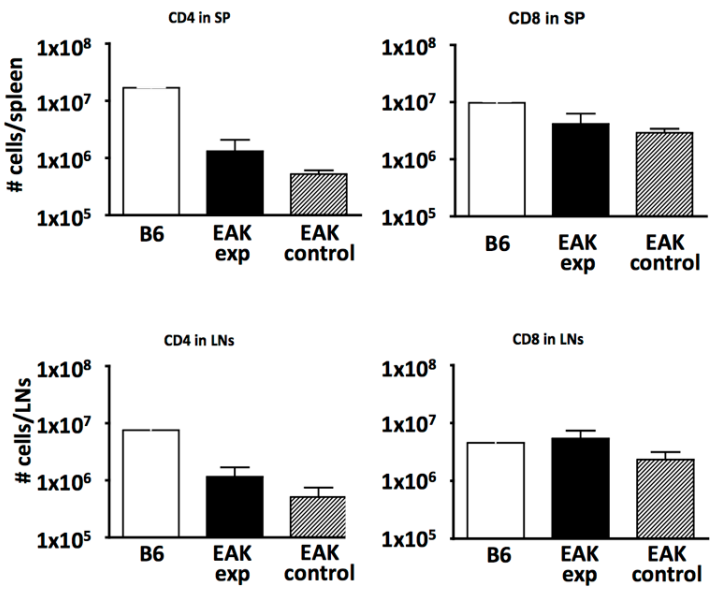

E

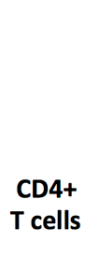

CD8+
T cells
B
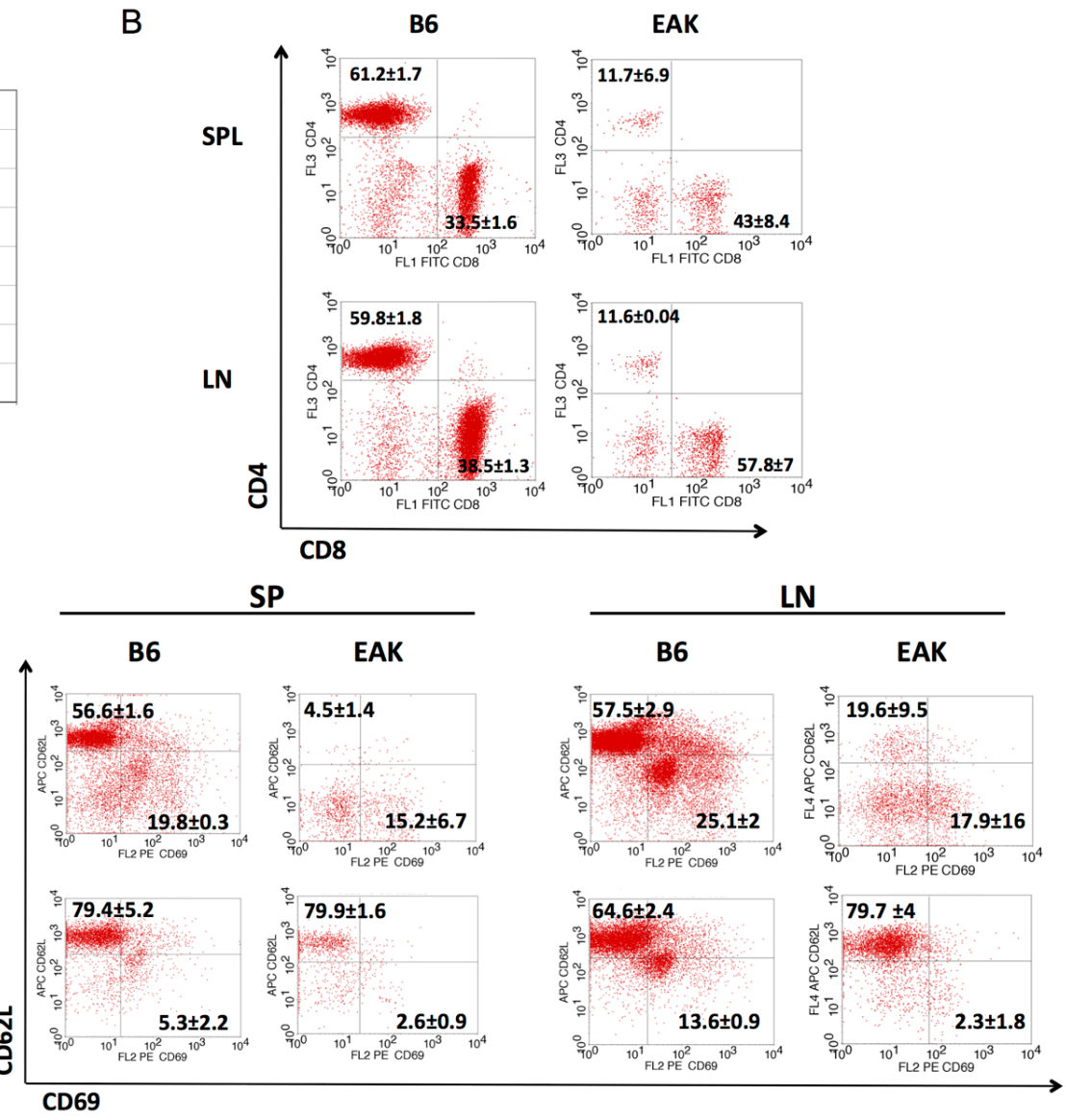

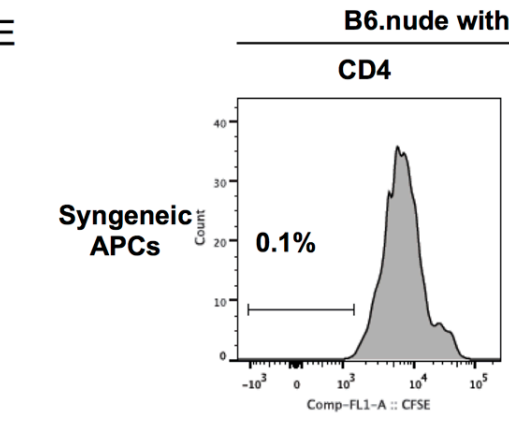

CD8
TEC/EAK thymus
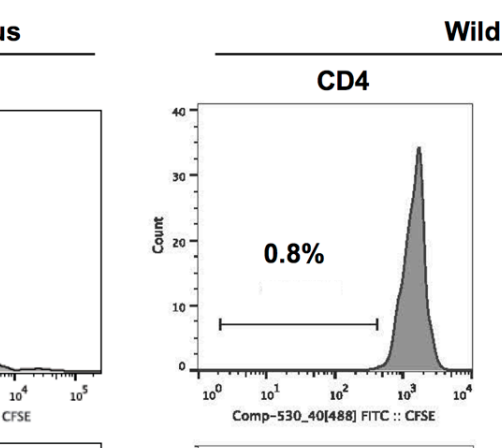

Wild type B6
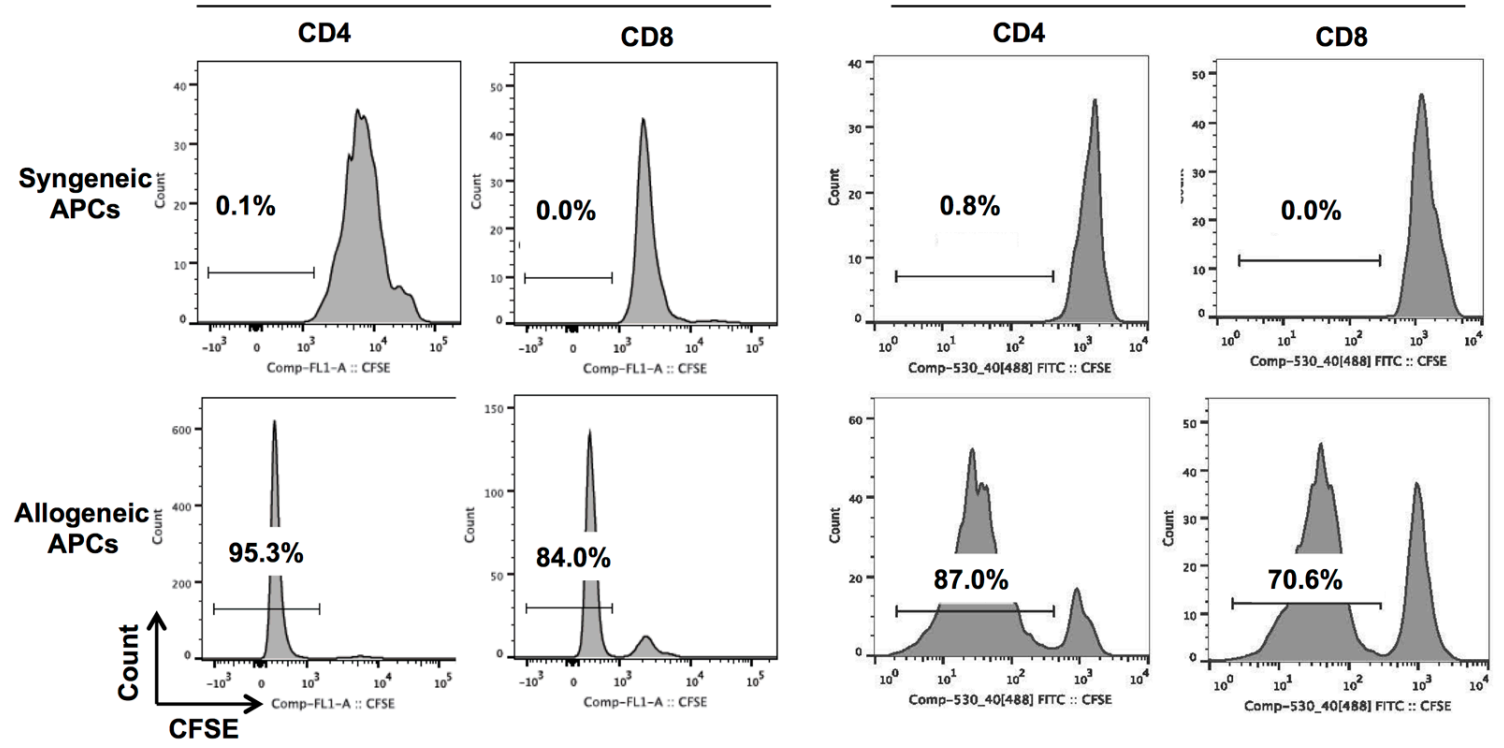